\title{
Highly sensitive optical microresonator sensors for photoacoustic imaging
}

Jing Li, Alaric Taylor, loannis Papakonstantinou, Edward Zhang, Paul Beard

Jing Li, Alaric Taylor, loannis Papakonstantinou, Edward Zhang, Paul Beard, "Highly sensitive optical microresonator sensors for photoacoustic imaging," Proc. SPIE 8943, Photons Plus Ultrasound: Imaging and Sensing 2014, 89430C (13 March 2014); doi: 10.1117/12.2037015

SPIE. Event: SPIE BiOS, 2014, San Francisco, California, United States 


\title{
Highly sensitive optical microresonator sensors for photoacoustic imaging
}

\author{
Jing Li*a, Alaric Taylor ${ }^{\mathrm{b}}$, Ioannis Papakonstantinou ${ }^{\mathrm{b}}$, Edward Zhang $^{\mathrm{a}}$ and Paul Beard ${ }^{\mathrm{a}}$ \\ ${ }^{a}$ Department of Medical Physics and Bioengineering, ${ }^{b}$ Department of Electronic and Electrical, \\ University College London, Gower Street, London WC1E 6BT, UK
}

\begin{abstract}
We present novel concave Fabry Perot (FP) sensor arrays for photoacoustic imaging which were fabricated using a highprecision inkjet printing approach to produce the cavity and employed physical vapor deposition to form the dielectric mirrors. Our concave FP cavity design provides excellent beam confinement within the cavity enabling high finesse and thus high sensitivity to be achieved. Two such concave sensors are evaluated in terms of their sensitivity and acoustic bandwidth. A $66 \mu \mathrm{m}$ thick concave sensor is shown to provide a noise equivalent pressure (NEP) of $85 \mathrm{~Pa}$ and an acoustic bandwidth of $16 \mathrm{MHz}$, and can potentially be used as a sensitive broadband sensor for superficial imaging. A $250 \mu \mathrm{m}$ thick sensor with an NEP of $12 \mathrm{~Pa}$ and acoustic bandwidth of $4 \mathrm{MHz}$ was also developed for deep tissue imaging applications.
\end{abstract}

Keywords: Photoacoustics, non-invasive imaging, deep tissue imaging, Fabry Perot sensor, sensor array, concave cavity, ultrasound

\section{INTRODUCTION}

Photoacoustic imaging is a non-invasive biomedical imaging modality based on the detection of laser induced ultrasound waves[1]. As a hybrid modality, it takes advantage of the high-contrast and spectroscopic-based specificity of optical imaging and the high spatial resolution of ultrasound imaging. Planar Fabry Perot (FP) ultrasound sensing etalons have proved to be extremely effective for superficial $(<1 \mathrm{~cm})$ photoacoustic imaging applications [2 -5$]$. To achieve the acoustically small element sizes ( $\sim$ tens of microns) required for high resolution tomographic imaging, the etalon is typically illuminated with a focused laser beam. However, the divergence of the beam causes beam walk-off, fundamentally limiting the etalon finesse and thus the sensor sensitivity. In essence, the problem is one of insufficient optical confinement and as a consequence, it has proved challenging to achieve imaging penetration depths greater than approximately $10 \mathrm{~mm}$ with planar FP ultrasound sensors. In this paper, we present a novel concave sensor design on planar substrate following previous work on fiber optic endoscopic probes [6], the geometry of which matches the wavefront of the interrogation laser beam. Due to the much stronger optical confinement provided by this approach, a significantly higher finesse yielding a sensitivity that significantly exceeds that of the most sensitive planar FP sensor is achievable.

*j.li@ucl.ac.uk; phone +44 2076790311

Photons Plus Ultrasound: Imaging and Sensing 2014, edited by Alexander A. Oraevsky, Lihong V. Wang, Proc. of SPIE Vol. 8943, 89430C · C 2014 SPIE · CCC code: 1605-7422/14/\$18 · doi: 10.1117/12.2037015 


\section{OVERVIEW OF FP ULTRASOUND SENSOR}

\subsection{Transduction mechanism}

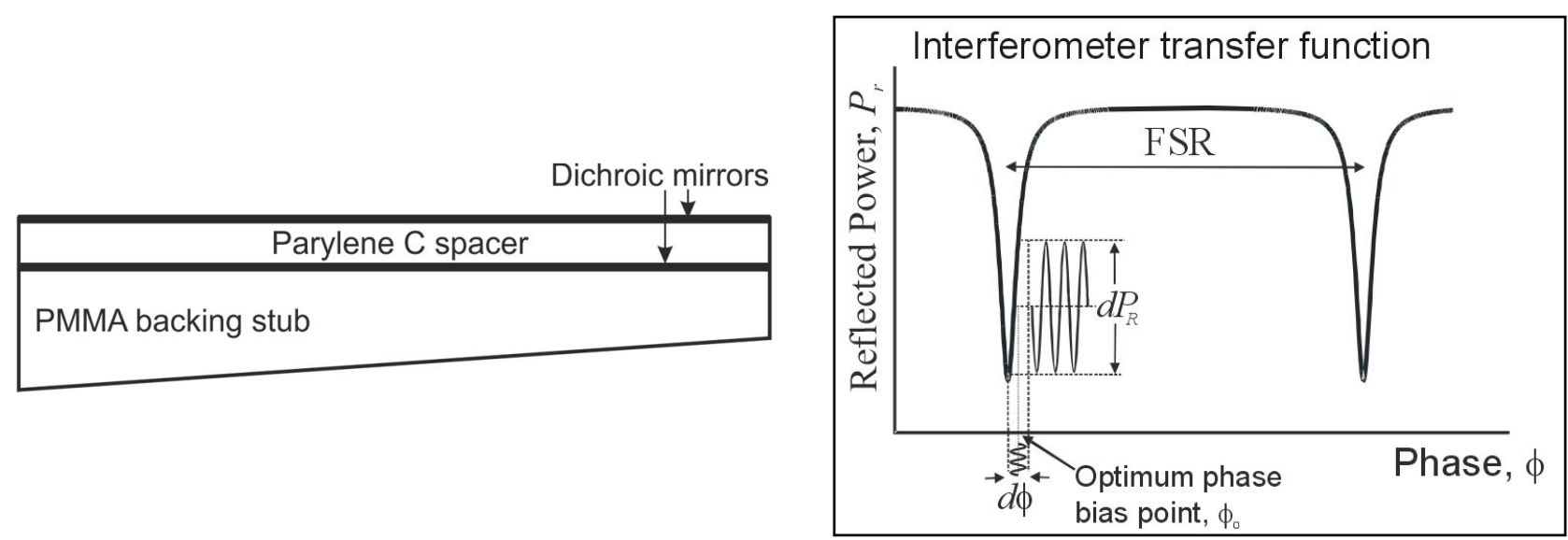

(a)

(b)

Figure 1. (a) Schematic of an existing planar FP sensor. (b) Interferometer transfer function showing reflected optical power as a function of optical phase. The operation at optimum phase bias point $\phi_{0}$ for the linear detection of a small acoustically induced phase modulation $d \phi$ is illustrated.

A schematic of an existing planar FP sensor is shown in Figure 1 (a) [3]. It consists of a thin $(l=20-60 \mu \mathrm{m})$ polymer spacer sandwiched between a pair of dichroic mirrors, forming a FP cavity, which is bonded to a transparent backing. An interrogation laser beam is incident on the sensor head and is multiply reflected between the two mirrors before exiting the cavity. An incident acoustic wave modulates the thickness of the polymer spacer, producing an optical phase shift of the light reflected from the two mirrors and resulting in a corresponding modulation of the reflected optical power of the interrogation laser beam. The variation in the reflected optical power from a FP sensor as a function of optical phase shift $\phi$ is defined as the phase interferometer transfer function (ITF) where $\phi$ is given by:

$$
\phi=\frac{4 \pi n l}{\lambda}
$$

where $n$ and $l$ are the refractive index and thickness of the polymer spacer respectively, and $\lambda$ is the wavelength of the interrogation laser beam. An example of the ITF is shown in Figure 1 (b). In order to make an acoustic measurement, the laser wavelength is adjusted to the optimal phase bias point $\phi_{0}$ that corresponds to the maximum slope of the ITF. Under this condition, maximal sensitivity can be achieved, and the FP sensor is said to be optimally biased [3]. If the acoustically induced phase shift $d \phi$ is small, it can be assumed to be linearly converted to a corresponding change in the reflected optical power, $d P_{R}$.

\subsection{Sensitivity}

The sensitivity of the FP sensor is defined as the change in reflected optical power per unit change of acoustic pressure. It can be divided into two parts - the acoustic phase sensitivity and the optical sensitivity [7].

The acoustic phase sensitivity represents the magnitude of the optical phase shift produced per unit acoustic pressure. It is a measure of acoustically-induced change in the optical thickness of the polymer spacer, resulting from change in both physical thickness and refractive index, and can be written as [7]:

$$
A_{S}=\frac{d \phi}{d p}=\frac{4 \pi}{\lambda}\left(n \frac{d l}{d p}+l \frac{d n}{d p}\right) P_{I}(k),
$$

where $p$ is the acoustic pressure, $n, l$ and $\lambda$ are the same as defined in Equation (1), $d l$ and $d n$ are a small change in the spacer thickness and a small change in the refractive index, respectively, $k=2 \pi / \lambda_{a}$ is the acoustic wavenumber, with $\lambda_{a}$ being the acoustic wavelength. $P_{I}(k)$ is a frequency-dependent modifying term that accounts for the spatial variation 
of stress within the spacer that occurs when an acoustic wave is incident on the sensor [2]. In the low frequency limit where $\lambda_{a} \gg l$, and under the assumption that the acoustic impedance mismatch between the spacer and the surrounding fluid is small, $P_{I}(k)$ is dominated by the acoustic properties of the backing material. For the polymer spacer used in the sensors described in this study, the elasto-optic effect can be neglected. Equation (2) can therefore be written as [7]:

$$
A_{S}=\frac{d \phi}{d p}=\frac{4 \pi n}{\lambda} \frac{l}{E} P_{I}(k),
$$

where $E$ is the Young's modulus of the spacer. As can be seen from Equation (3), the acoustic phase sensitivity scales linearly with the thickness of the spacer, albeit with a commensurate reduction in acoustic bandwidth which scales inversely with thickness $[2,8]$.

The optical sensitivity describes the optical power modulation per unit phase shift at the phase bias point and is defined as [7]:

$$
I_{S}=\frac{d P_{R}}{d p}
$$

which is the first derivative of the ITF at the phase bias point. In general, $I_{S}$ increases as the finesse and fringe visibility increase.

The overall detection sensitivity, $S$, is the product of the acoustic phase sensitivity and the optical sensitivity.

\section{CONCAVE FP SENSOR}

\subsection{Sensitivity enhancement over planar FP sensor}

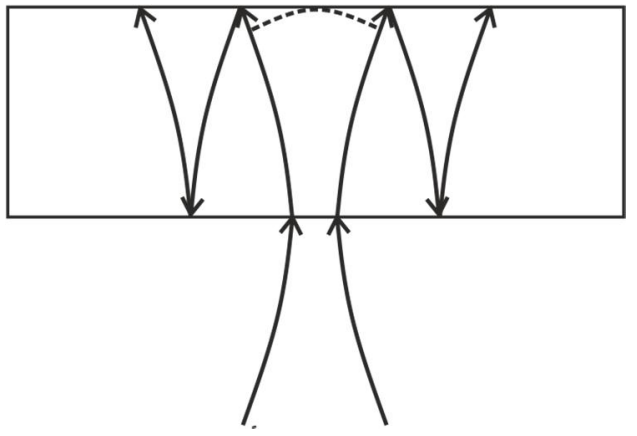

(a)

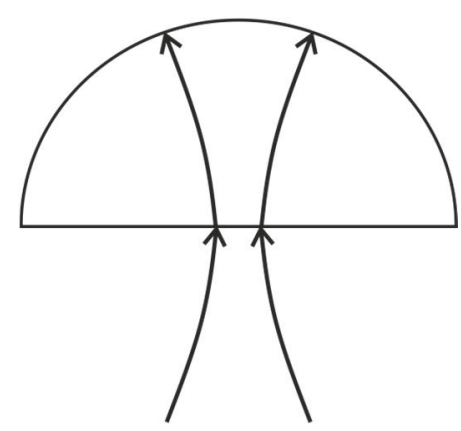

(b)

Figure 2. Schmatics of the beam progation within (a) a planar FP cavity and (b) a concave cavity whose geometry matches the wavefront of the incident laser beam.

A three-dimensional imaging system based on a planar FP ultrasound sensor was previously developed by Zhang et al. [3]. In their system, a focused laser beam was used to interrogate the sensor in order to minimize the effective acoustic element size. However, due to the mismatch between the wavefront of the divergent beam and the planar mirrors, phase dispersion and beam walk-off was significant after several round trips within the FP cavity, as illustrated in Figure 2 (a). This degrades the finesse and fringe visibility and therefore the optical sensitivity of the FP sensor. The deterioration of the optical sensitivity is even more significant for thick planar sensors thus limiting the maximum thickness to approximately $50 \mu \mathrm{m}$ for beam radii of a few tens of microns. In both cases, improved beam confinement within the cavity is needed. This can be achieved by using a planar-concave cavity configuration. 
A focused single mode interrogation laser beam can be approximated by a propagating Gaussian beam, with its wavefront curvature at a distance of $z$ from the minimum beam waist given by [9]:

$$
R(z)=z\left[1+\left(\frac{\pi \omega_{0}^{2}}{\lambda z}\right)^{2}\right],
$$

where $\omega_{0}$ is the minimum beam waist and $\lambda$ is the wavelength. The best beam confinement can be achieved by matching the curvature of the cavity mirrors with the wavefront of the Gaussian beam. In the planar-concave configuration, the planar mirror is located at the minimum beam waist, where the wavefront curvature is infinite. The concave mirror has a radius of curvature that matches the wavefront curvature at a location $l$ given by Equation (5), with $l$ being the cavity length, as shown in Figure 2 (b). Theoretically, under this condition, the beam can exactly replicate itself after each reflection from the cavity mirrors offering the prospect of achieving higher finesse and fringe visibility than possible with a planar FP sensor.

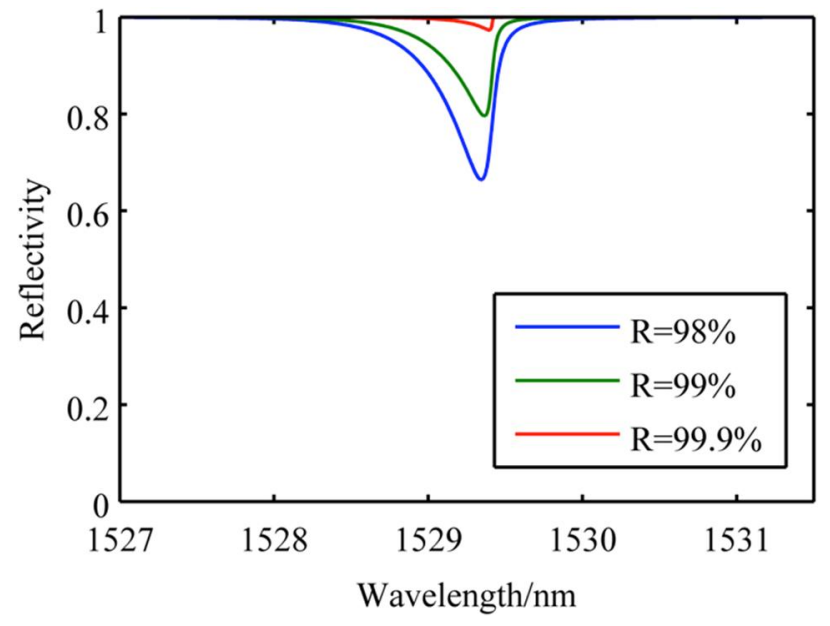

(a)

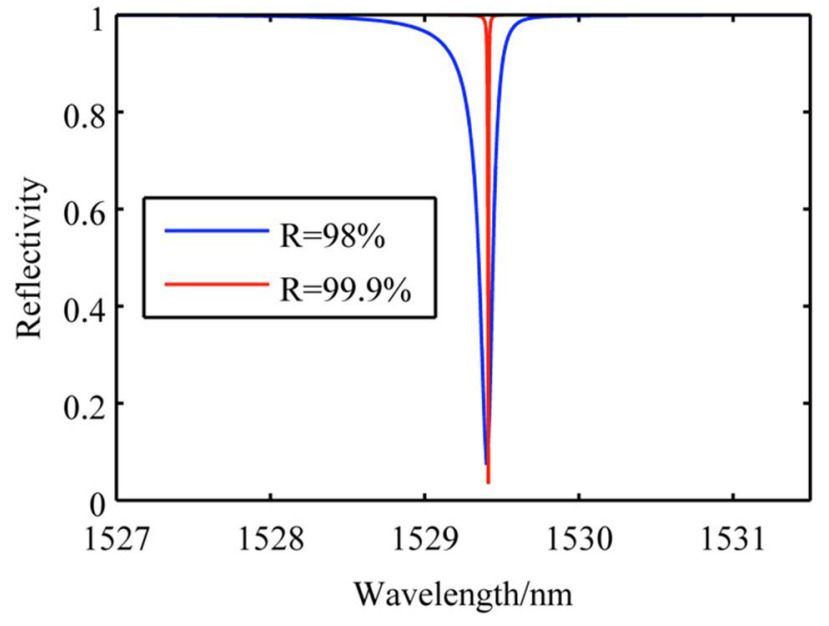

(b)

Figure 3. (a) Simulated ITFs of a planar sensor of thickness $50 \mu \mathrm{m}$, with incident beam waist of $25 \mu \mathrm{m}$, for mirror reflectivity of $98 \%($ Finesse $=44$, visibility $=0.2), 99 \%($ Finesse $=56$, visibility $=0.11)$, and $99.9 \%($ Finesse $=110$, visibility $=0.01$ ), respectively. (b) Simulated ITFs of a concave sensor of thickness $50 \mu \mathrm{m}$, with incident beam waist of 25 $\mu \mathrm{m}$, for mirror reflectivity of $98 \%$ (Finesse $=145$, visibility $=0.9$ ) and $99.9 \%$ (Finesse $=2970$, visibility $=0.93$ ), respectively.

To compare the optical sensitivity of the planar and concave sensors, the ITFs of two sensors with the same thickness $(l$ $=50 \mu \mathrm{m})$ and incident beam waist $\left(\omega_{0}=25 \mu \mathrm{m}\right)$ and a few values of mirror reflectivity, $R$, were simulated using a numerical model based on the tracing of Gaussian beam propagation within a planar or a concave FP cavity, and the results are shown in Figure 3 (a) and (b). For a planar sensor with $R=98 \%$, the finesse and fringe visibility are equal to 44 and 0.2 , respectively, while the concave sensor with the same $R$ has finesse and visibility of 145 and 0.9 , respectively. One should therefore expect at least a threefold increase of optical sensitivity when using the concave sensor. In principle, the finesse of the planar cavity can be increased by using mirrors with higher reflectivity. However, as shown in Figure 3 (a), this results in a rapid decrease in visibility. As $R$ increases from $98 \%$ to $99 \%$ and then to $99.9 \%$, the finesse increases from 44 to 56 and then to 110 , but the visibility decreases from 0.2 to 0.11 and then to 0.01 at the same time. In contrast, for the concave sensor, increasing the mirror reflectivity increases the finesse, without sacrificing the visibility, as shown in Figure 3 (b). 


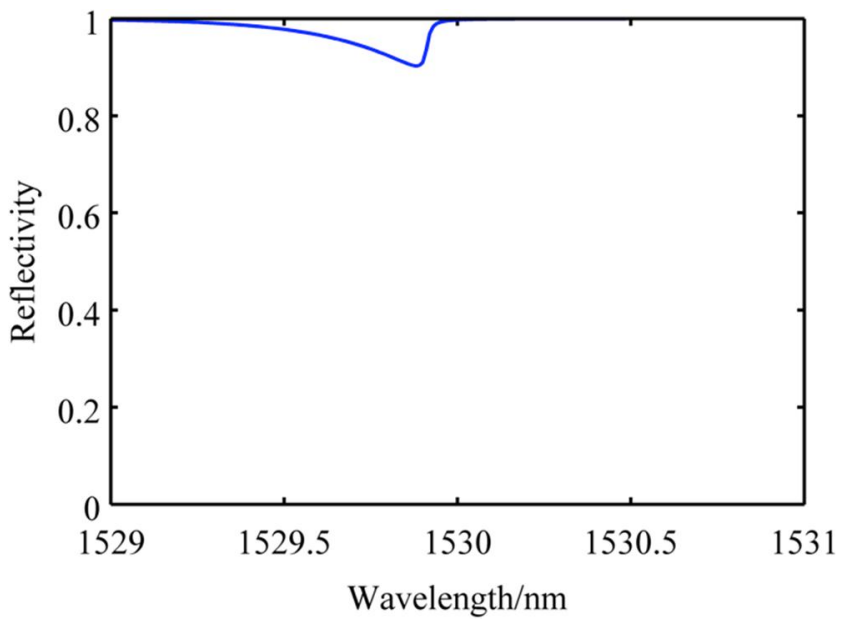

(a)

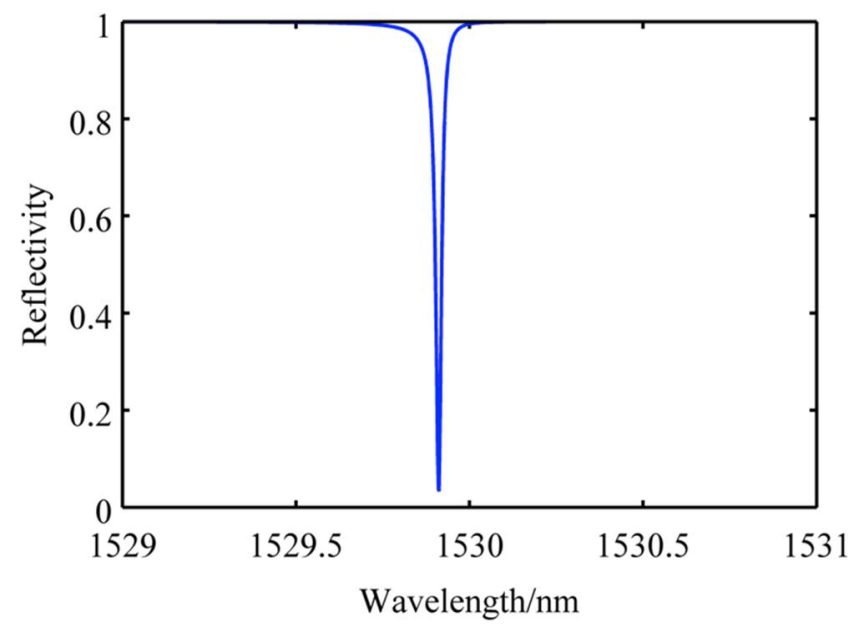

(b)

Figure 4. (a) Simulated ITF of a planar sensor of thickness $250 \mu \mathrm{m}$, with incident beam waist of $25 \mu \mathrm{m}$ and mirror reflectivity of $98 \%$ (Finesse $=13$, visibility $=0.05$ ). (b) Simulated ITF of a concave sensor of thickness $250 \mu \mathrm{m}$, with incident beam waist of $25 \mu \mathrm{m}$ and mirror reflectivity of $98 \%($ Finesse $=152$, visibility $=0.95)$.

As discussed in Section 2.2, the detection sensitivity comprises both the optical sensitivity and the acoustic phase sensitivity. In principle, further increase of overall sensitivity can be achieved by using a thicker sensor if the same optical sensitivity is maintained. Of course an increase in sensor thickness is always accompanied by reduced detection bandwidth. However, this reduced bandwidth can sometimes be tolerated in deep tissue imaging applications such as clinical breast imaging [10 - 12], where frequency-dependent acoustic attenuation by the tissue results in the high frequency components of the acoustic wave being preferentially attenuated.

Increasing the thickness of a planar FP sensor without compromising the finesse and visibility can be challenging due to significant phase dispersion and beam walk-off. On the other hand, a carefully designed concave sensor can still offer good optical sensitivity owing to better beam confinement. Figure 4 (a) and (b) illustrate this by comparing the simulated ITFs of a planar sensor and a concave sensor with the same thickness of $250 \mu \mathrm{m}$, respectively. For $R=98 \%$ and $\omega_{0}=25$ $\mu \mathrm{m}$, the finesse is only 13 for the planar sensor, while it is as high as 152 for the concave sensor. The visibility of the concave sensor is also much better than its planar counterpart.

\subsection{Fabrication method}

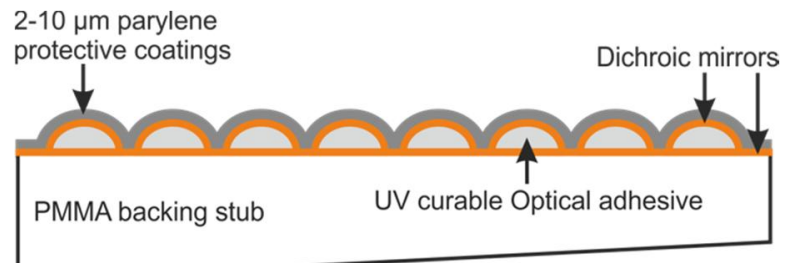

(a)

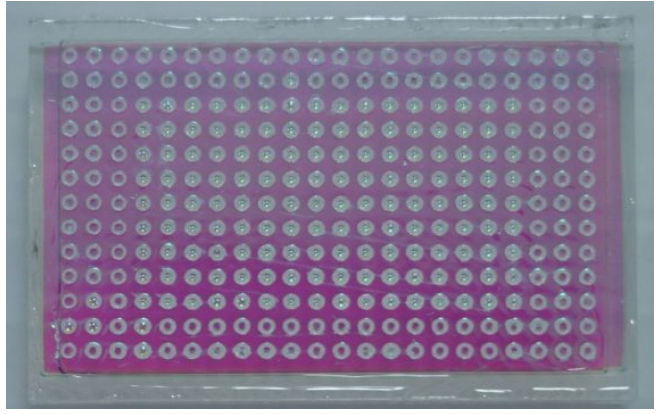

(b)

Figure 5. (a) Schematic and (b) photograph of a concave sensor head. 
A schematic and a photo of a concave sensor head are shown in Figure 5 (a) and (b), respectively. The first dichroic mirror is deposited onto a wedged polymethylmethacrylate (PMMA) backing stub by sputtering a stack of alternate $\lambda / 4$ thick layers of $\mathrm{ZnS}$ and $\mathrm{Na}_{3} \mathrm{AlF}_{6}$. The nominal reflectivity of the mirror is $98 \%$. On top of the dielectric coating are the domes made of a UV curable optical adhesive (Norland 81) of refractive index $n=1.56$, fabricated using an inkjet printing method. This allows automatic printing of dome arrays with a wide range of geometries and sub-picoliter volume accuracy. After being fully cured by a UV lamp, a second dichroic mirror coating of an identical design to the first is deposited onto the formed domes. Finally, a $2-10 \mu \mathrm{m}$ thick Parylene $\mathrm{C}$ barrier coating is deposited over the entire structure to protect the external dichroic mirror from damage due to abrasion or water ingress. Using this fabrication method, domes with height in the range of $40-400 \mu \mathrm{m}$ and diameter in the range of $50-2000 \mu \mathrm{m}$ have been made.

\section{SENSOR CHARACTERIZATION}

\subsection{Experimental setup}

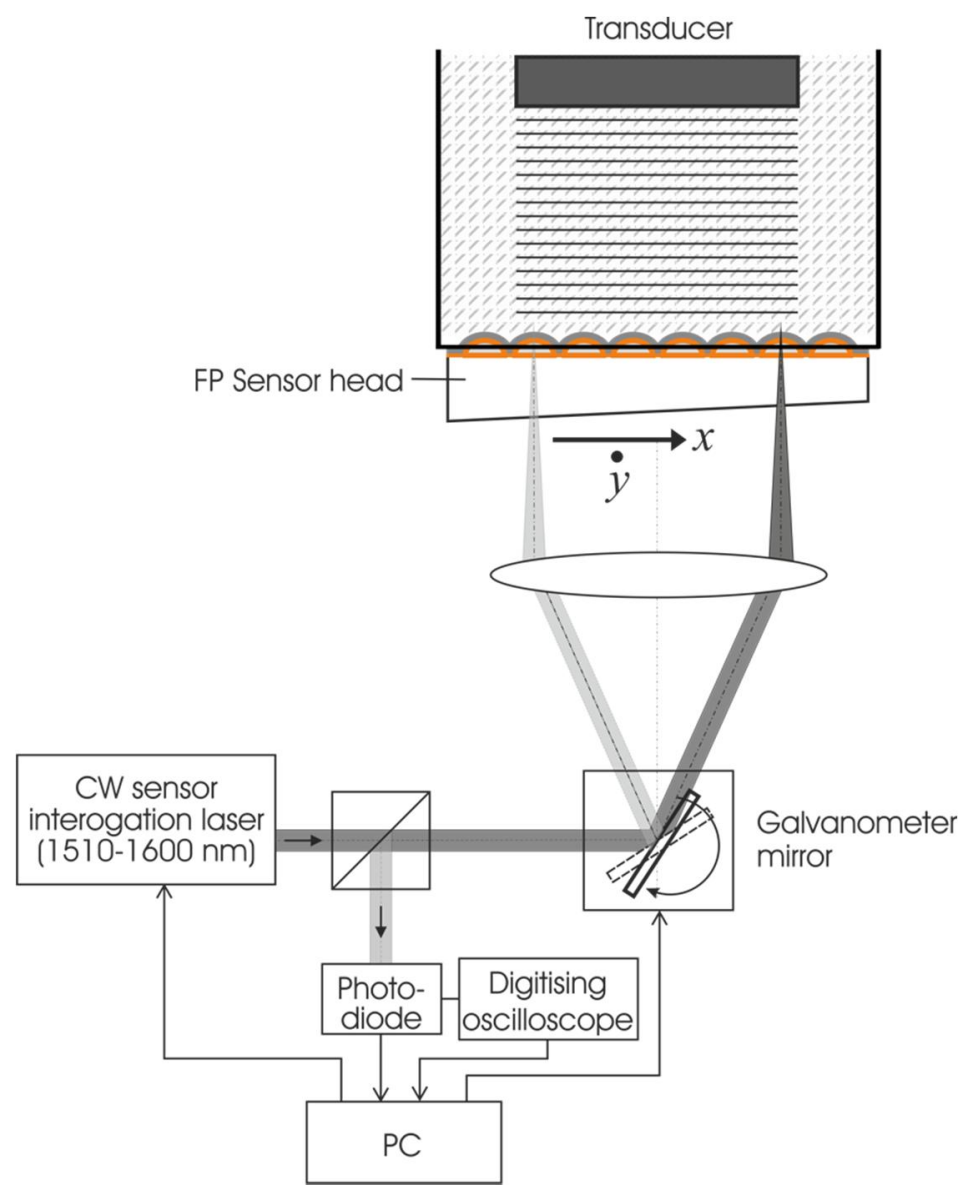

Figure 6. Schematic of the sensor characterization setup.

A schematic of the experimental setup used for sensor characterization is shown in Figure 6. The interrogation laser source is an external cavity laser, which can be tuned over the wavelength range of $1510-1600 \mathrm{~nm}$. The collimated output beam of the laser is steered by a pair of high-speed precision galvanometer mirrors in both $x$ and $y$ directions. The FP sensor head is situated in the focal plane of the scan lens. The beam waist at the focus is approximately $10 \mu \mathrm{m}$. For each concave sensor under investigation, the sensor is optimally biased by tuning the interrogation laser wavelength to 
the point of maximum slope on the ITF to achieve the highest optical sensitivity. Planar PZT transducers with different bandwidths depending on the specific applications are used to generate acoustic waves propagating towards the sensor. The beam reflected from the sensor is then directed onto a photodiode and measured by a digital sampling oscilloscope. The entire system is fully automated with the interrogation laser, the galvanometer, and the data acquisition hardware all controlled by a PC.

\subsection{Results and discussion}

The performance of two types of concave sensor has been assessed in terms of ITF, noise-equivalent-pressure (NEP) and frequency response. The ITF is measured by scanning the wavelength of the interrogation laser across the free spectral range (FSR) and recording the normalized reflectivity. The NEP is defined as the acoustic pressure that provides a system signal-to-noise ratio (SNR) of unity in the low frequency limit where the acoustic wavelength is much larger than the sensor thickness. The NEP thus represents the minimum acoustic pressure that can be detected by the system and is given by [3]:

$$
\mathrm{NEP}=\frac{N}{S_{0}},
$$

where $S_{0}$ is the sensor sensitivity and is defined as the reflected optical power modulation per unit acoustic pressure, and $N$ is the minimum detectable optical power over a specified measurement bandwidth.

In practice, $S_{0}$ is determined by measuring the output voltage of the photodiode in response to an acoustic signal of known amplitude produced by a pre-calibrated pulsed 3.5 MHz planar PZT transducer. $N$ is taken as three times the standard deviation of the noise voltage in order to estimate the peak value. The measurement bandwidth was $20 \mathrm{MHz}$.

To measure the frequency response, a broadband acoustic pulse generated by a $20 \mathrm{MHz}$ planar transducer was detected first with a $25 \mu \mathrm{m}$ thick planar FP sensor of known frequency response characteristics that acted as a reference sensor and then by the concave sensors. The time-domain waveforms were then Fourier transformed and the frequency response of the concave sensor was obtained by dividing the acoustic spectrum of the sensor under test by that of the reference sensor [3].

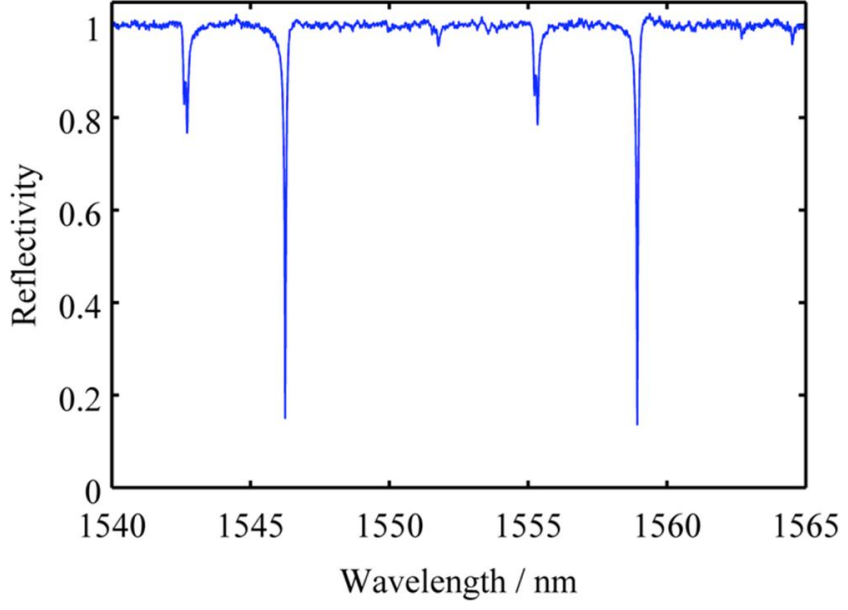

(a)

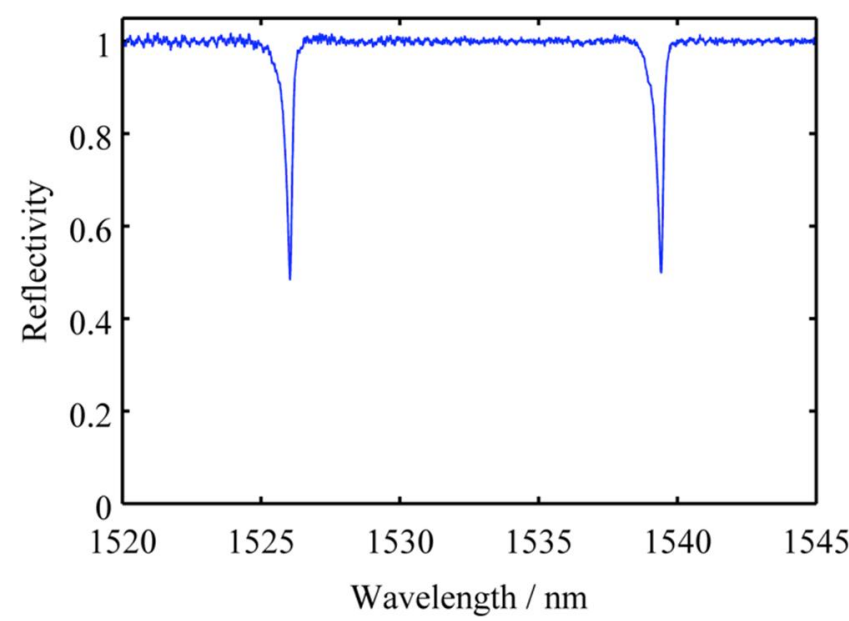

(d) 


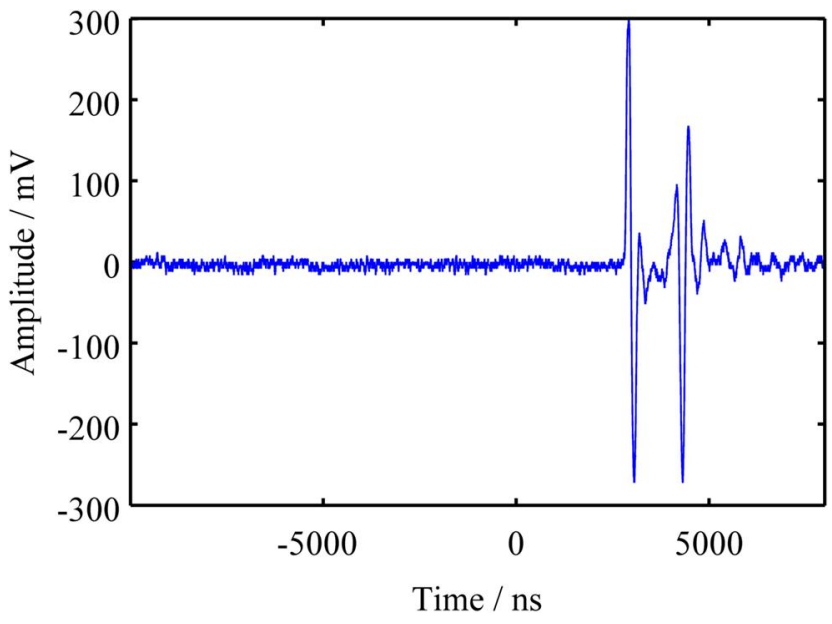

(b)

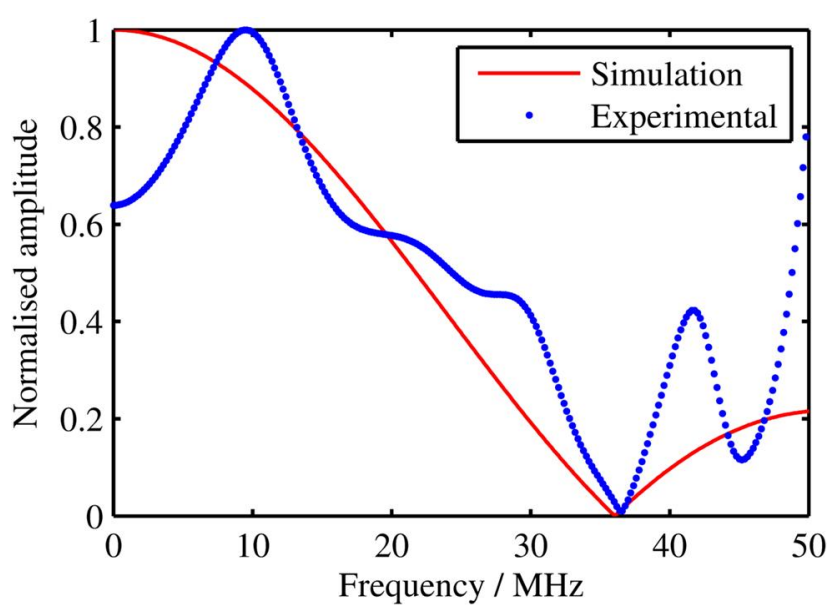

(c)

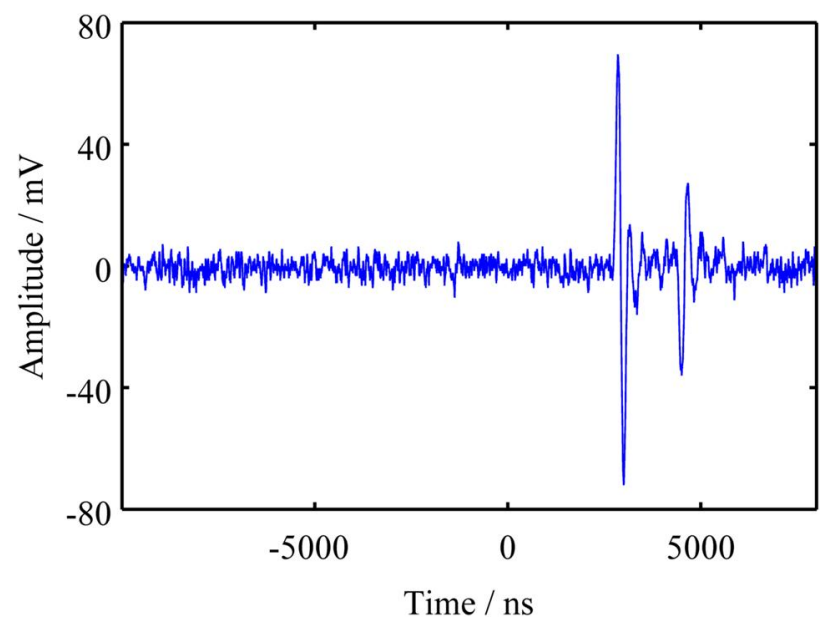

(e)

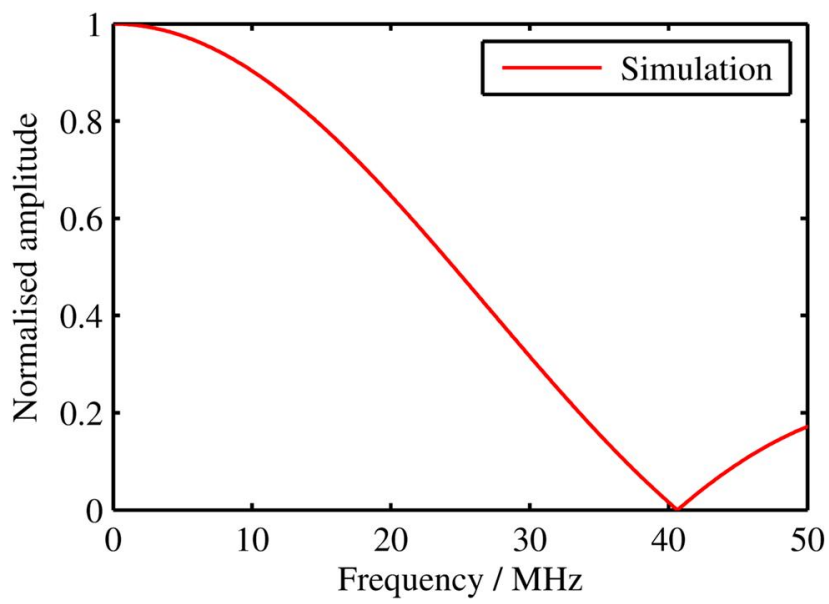

(f)

Figure 7. (a) ITF, (b) time-domain waveform, and (c) frequency response of a concave sensor of thickness $66 \mu \mathrm{m}$ and diameter $420 \mu \mathrm{m}$. Finesse $=218$, visibility $=0.76, \mathrm{NEP}=85 \mathrm{~Pa}$ (measurement bandwidth $=20 \mathrm{MHz}), f_{-3 d B}=16 \mathrm{MHz}$. $(\mathrm{d})$ ITF, (e) time-domain waveform, and (f) frequency response of a planar sensor of thickness $56 \mu \mathrm{m}$. Finesse $=60$, visibility $=$ $0.35, \mathrm{NEP}=370 \mathrm{~Pa}$ (measurement bandwidth $=20 \mathrm{MHz}$ ), $f_{-3 d B}=18 \mathrm{MHz}$. The simulated acoustic frequency responses were obtained using the model described in reference 2 .

Figure 7 (a) - (c) shows the ITF, the time-domain waveform and the frequency response of a concave sensor of thickness $66 \mu \mathrm{m}$ and diameter $420 \mu \mathrm{m}$. As a comparison, the results for a planar sensor of similar thickness $(56 \mu \mathrm{m})$ and thus similar bandwidth are also shown in Figure 7 (d) - (f). Note that the secondary reflectivity minima in Figure 7 (a) are due to a slight mismatch of the beam wavefront and the mirror curvature, resulting in excitation of an additional transverse mode. Very narrow resonance peaks have been observed for the concave sensor, corresponding to a finesse as high as 218 , which is more than three times the value of the planar sensor (finesse $=60$ ). The visibility of the concave sensor is also much better than the planar one. The NEP of the concave sensor is $85 \mathrm{~Pa}$, compared to $370 \mathrm{~Pa}$ for the planar sensor. In both cases the measurement bandwidth was $20 \mathrm{MHz}$. The increased sensitivity of the concave sensor can be readily observed by comparing the SNR in the time-domain waveforms to that of the planar sensor, as shown in Figure 7 (b) and (e). With a $-3 \mathrm{~dB}$ bandwidth of approximately $16 \mathrm{MHz}$, this concave sensor can be potentially used as a sensitive broadband sensor. 


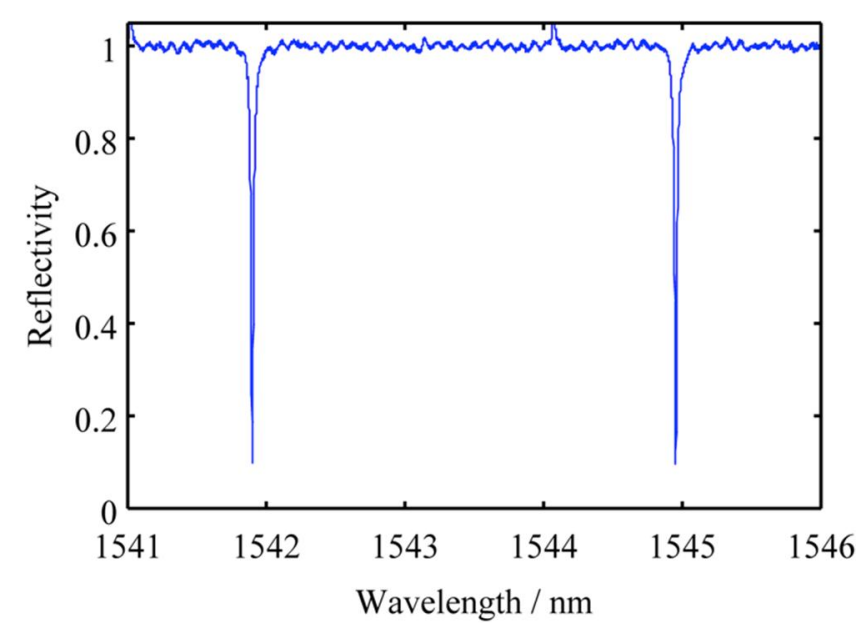

(a)

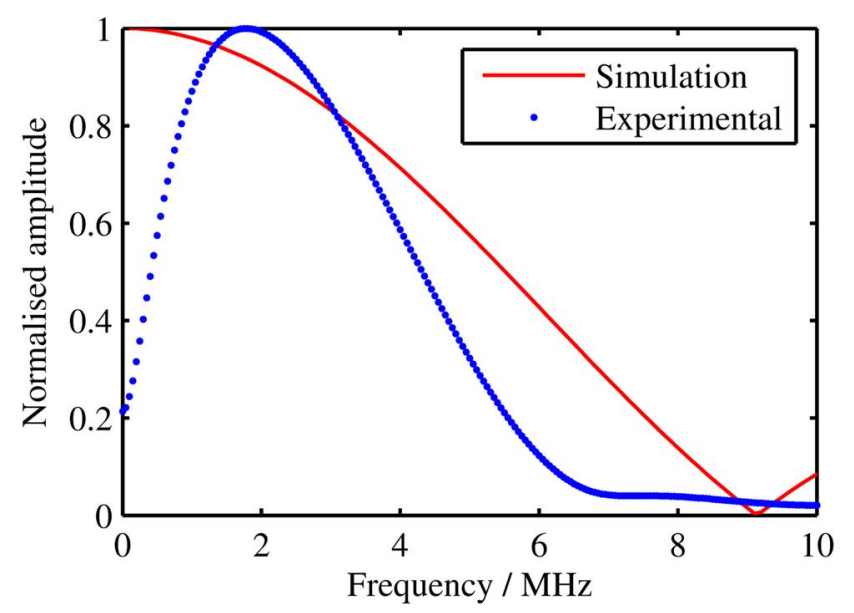

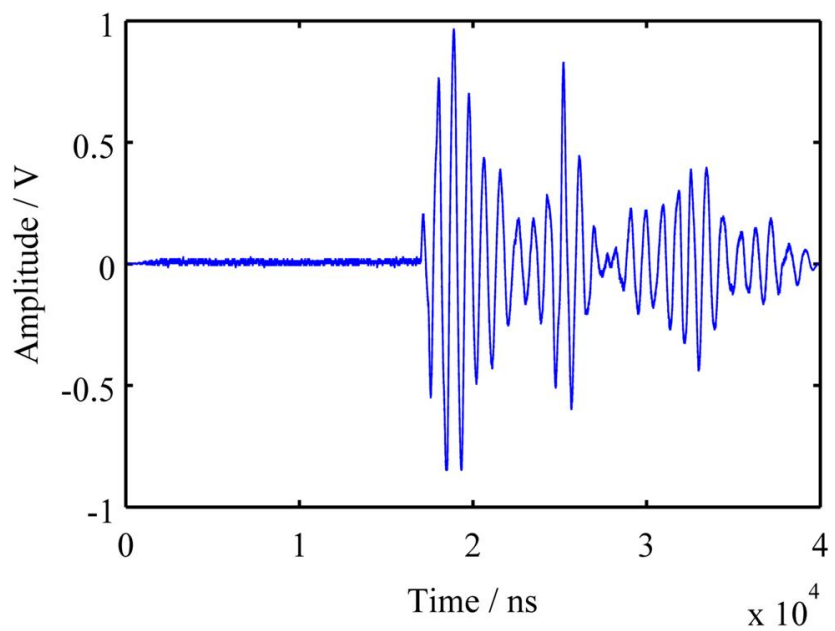

(b)

(c)

Figure 8. (a) ITF, (b) time-domain waveform, and (c) frequency response of a concave sensor of thickness $250 \mu \mathrm{m}$ and diameter $1100 \mu \mathrm{m}$. Finesse $=168$, visibility $=0.86, \mathrm{NEP}=85 \mathrm{~Pa}$ (measurement bandwidth $=12 \mathrm{MHz}), f_{-3 d B}=4 \mathrm{MHz}$. The simulated acoustic frequency responses were obtained using the model described in reference 2 .

As discussed earlier, thick sensors might be needed for deep tissue imaging, where high sensitivity is required for the detection of weak acoustic signals, and narrow bandwidths can be tolerated [8, 11\&12]. Here a $250 \mu \mathrm{m}$ thick concave sensor with diameter of $1100 \mu \mathrm{m}$ has been made and investigated. The results are shown in Figure 8 (a) - (c). As can be seen from the ITF in Figure 8 (a), high finesse (168) and good visibility (0.86) are maintained, despite the thickness of the sensor. The excellent optical sensitivity together with extra gain in acoustic phase sensitivity from increased sensor thickness makes this sensor extremely sensitive, as evidenced by the high SNR in the waveform shown in Figure 8 (b). The NEP is as low as $12 \mathrm{~Pa}$ within a $20 \mathrm{MHz}$ bandwidth region, which is reduced by nearly 30 times from that of the 56 $\mu \mathrm{m}$ planar sensor discussed above. The $-3 \mathrm{~dB}$ bandwidth of this concave sensor is approximately $4 \mathrm{MHz}$, making it potentially suitable for imaging tissue to depths of several $\mathrm{cm}$. 


\section{CONCLUSIONS}

This work has shown that highly sensitive acoustic sensors can be achieved with the use of a concave FP cavity geometry. If the radii of curvature of the cavity mirrors match the wavefront of the interrogation laser beam, good beam confinement within the cavity can be achieved, resulting in significant sensitivity enhancement compared with the previously used planar FP sensor. An automated fabrication method has been used to make concave sensor arrays with high precision. The performance of two typical concave sensors has been investigated. The $66 \mu \mathrm{m}$ thick sensor with a $3 \mathrm{~dB}$ bandwidth of $16 \mathrm{MHz}$ and NEP of $85 \mathrm{~Pa}$ (over a $20 \mathrm{MHz}$ measurement bandwidth) can potentially be used as a sensitive broadband sensor. The $250 \mu \mathrm{m}$ thick sensor with a $-3 \mathrm{~dB}$ bandwidth of $4 \mathrm{MHz}$ and NEP of $12 \mathrm{~Pa}$ (over a 20 $\mathrm{MHz}$ measurement bandwidth) could serve as an ultra-sensitive detector for clinical breast imaging and other deep tissue imaging applications.

\section{REFERENCES}

[1] Beard, P.C, "Biomedical photoacoustic imaging," Interface Focus 1(4), 602-631 (2011).

[2] Beard, P.C., Perennes, F., and Mills, T.N., "Transduction mechanisms of the Fabry-Perot polymer film sensing concept for wideband ultrasound detection," IEEE Transactions on Ultrasonics, Ferroelectrics and Frequency control 46(6), 1575-1582 (1999).

[3] Zhang, E.Z., and Beard, P.C., "Broadband ultrasound field mapping system using a wavelength tuned, optically scanned focused laser beam to address a Fabry Perot polymer film sensor," IEEE Transactions on Ultrasonics, Ferroelectrics, and Frequency Control 53, 1330-1338 (2006).

[4] Zhang, E.Z, Laufer, J., and Beard, P.C, "Backward-mode multiwavelength photoacoustic scanner using a planar Fabry-Perot polymer film ultrasound sensor for high-resolution three-dimensional imaging of biological tissues," Applied Optics 47, 561-577 (2008).

[5] Laufer, J., Johnson, P., Zhang, E.Z., Treeby, B., Cox, B., Pedley, B., \& Beard, P.C., "In vivo preclinical photoacoustic imaging of tumor vasculature development and therapy," Journal of Biomedical Optics, 17(5), 056016. (2012).

[6] Zhang, E.Z., and Beard, P.C., "A miniature all-optical photoacoustic imaging probe," Proc. of SPIE, Vol. 7899, paper 78991F (2011).

[7] Cox, B.T., and Beard, P.C., "The frequency-dependent directivity of a planar Fabry-Perot polymer film ultrasound sensor," IEEE Transactions on Ultrasonics, Ferroelectrics and Frequency control 54, 394-404 (2007).

[8] Allen, T.J., and Beard, P.C., "Optimising the detection parameters for deep tissue photoacoustic imaging," Proc. of SPIE 8223, paper 82230P (2012).

[9] Silfvast, W.T., [Laser Fundamentals], Cambridge University Press, (2008).

[10] Kruger, R.A., Lam, R.B., Reinecke, D.R., Del Rio, S.P., and Doyle, R.P., "Photoacoustic angiography of the breast," Medical Physics 37, 6096 (2010).

[11] Piras, D., Steenbergen, W., van Leeuwen, T.G., and Manohar, S.G., "Photoacoustic imaging of the breast using the twente photoacoustic mammoscope: present status and future perspectives," IEEE Journal of Selected Topics in Quantum Electronics 16, 730-739 (2010).

[12] Xia, W., Piras, D., van Hespen, J.C.G., et al., "An optimized ultrasound detector for photoacoustic breast tomography," Medical Physics 40, 032901 (2013). 